\title{
Estimación de la salinidad en suelos del delta del río Sinú en Colombia, mediante modelos de regresión lineal múltiple
}

\author{
Estimation of salinity in soils of river delta of Sinu in Colombia, \\ through of linear multiple regression models
}

\author{
Humberto Narváez M. ${ }^{1}$, Iván Bustamante Barrera ${ }^{1}$,Enrique Combatt C. $^{1 *}$
}

\begin{abstract}
RESUMEN
La estadística clásica y geoestadística son algunas de las herramientas para el análisis de la salinidad de suelos que contribuyen en la identificación de áreas afectadas, monitoreo de variaciones espacio-temporales y la formulación de estrategias de manejo. El objetivo del trabajo fue generar y validar modelos para estimar la conductividad eléctrica de los suelos ubicados en área de influencia de la desembocadura del río Sinú en Córdoba, Colombia. En el área de estudio de $82 \mathrm{~km}^{2}$ se instalaron 25 pozos en vértices principales con espaciamiento de $2 \mathrm{~km}$ entre sí y 14 en vértices auxiliares con espaciamiento de $1 \mathrm{~km}$ respecto de los principales. Próximo a cada vértice fueron instalados sistemáticamente 39 pozos de observación, en los cuales se colectaron muestras de suelos hasta los $50 \mathrm{~cm}$ de profundidad, durante 2002 a 2008. En las muestras de suelos se determinó la conductividad eléctrica (CE) de los iones $\mathrm{Ca}, \mathrm{Mg}, \mathrm{Na}, \mathrm{K}, \mathrm{HCO}_{3}, \mathrm{CO}_{3}$ y $\mathrm{SO}_{4}$ en pasta de saturación. Los datos obtenidos fueron sometidos a análisis de correlación simple, entre la CE y los cationes y aniones presentes en el extracto de saturación. La estimación de los modelos de CE se realizó por el método de regresión por pasos (The Stepwise regresión procedure) y su validación se realizó mediante la prueba chi-cuadrado entre valores de $\mathrm{CE}$ generados por los modelos y la obtenida en muestras de suelos. Los resultados indican una alta y significativa correlación entre la CE y los contenidos de iones $\mathrm{Cl}$, Na y Mg. Los modelos de CE calculados para las profundidades y periodos presentan $\mathrm{R}^{2}$ entre 0,84 y 0,96, indicando alto grado de confiabilidad para la estimación de la CE. De igual forma se determinó la contribución de cada ion sobre los modelos, donde fueron predominantes el $\mathrm{Cl}, \mathrm{Na}$ y $\mathrm{Mg}$.
\end{abstract}

Palabras clave: conductividad eléctrica, pasta de saturación, salinidad, modelos de regresión lineal múltiple.

\begin{abstract}
The classic statistics and geo-statistics are some of the analysis tools of soil saltiness that contribute to the identification of affected areas, monitoring the space-temporary variations, and the formulation of management strategies. The objective of this study was to generate and validate models for the electric conductivity estimation of the soil saturation extract (EC) on the area of influence of the Sinu River mouth in dry and rainy periods. The study area of $82 \mathrm{~km}^{2}$ was divided into four locations near the river mouth of the Sinu River, where 39 observation wells distributed in 25 wells in main vertices with spacing of $2 \mathrm{~km}$ apart and 14 auxiliary vertices with spacing $1 \mathrm{~km}$ of the main. For the sampling of groundwater two periods were chosen and corresponded to season of dry and rainy seasons. In the samples were evaluated the electrical conductivity (EC), $\mathrm{pH}, \mathrm{Ca}, \mathrm{Mg}, \mathrm{Na}, \mathrm{K}, \mathrm{HCO}_{3}, \mathrm{CO}_{3}$ and $\mathrm{SO}_{4}$. The data obtained were subjected to simple correlation analysis between the EC and the cations and anions present in the saturation extract. The estimating the models on CE was performed by the method of stepwise regression (The Stepwise regression procedure) and its validation was performed using the chi-square test between EC values generated by the models and that obtained in soil samples. The results indicate a highly significant correlation between the EC and the contents of Cl, Na and $\mathrm{Mg}$ ions. The CE models calculated for the depths and periods have $R^{2}$ between 0.84 and 0.96 , indicating a high degree of reliability for the estimation of the EC. Similarly, the contribution of each ion on the models was determined, which predominantly were $\mathrm{Cl}, \mathrm{Na}$ and $\mathrm{Mg}$.
\end{abstract}

Key words: electric conductivity, saltiness, models of multiple lineal regression, stepwise regression procedure.

\section{Introducción}

La salinidad, así como otras propiedades físicas y químicas del suelo, presentan variabilidad espacial y temporal en función de prácticas de manejo utilizadas, profundidad del nivel freático, permeabilidad del suelo, tasa de evapotranspiración y precipitación, salinidad del agua subterránea y de otros factores hidrogeológicos (D'Almeida et al., 2005). En regiones próximas a las costas marinas

\footnotetext{
$1 \quad$ Universidad de Córdoba, Carrera 6a \# 76-103. Telefax 574 7860255. Montería, Córdoba, Colombia. Córdoba.

* Autor por correspondencia: ecombatt@fca.edu.co; ecombatt@ correo.unicordoba.edu.co.
}

Fecha de Recepción: 16 Febrero, 2014.

Fecha de Aceptación: 3 Junio, 2014. 
en la desembocadura del río Sinú, la salinización de suelos puede deberse a la intrusión del agua de mar, que al estar sometida a la continua evaporación deposita sales en cantidades nocivas al desarrollo y crecimiento de las especies poco tolerables a estas condiciones. En estas condiciones edafológicas el incremento de la salinización reduce la expansión de la frontera agrícola, debido al ascenso capilar de aguas freáticas ricas en sales solubles, que se suman al aporte de iones que se originan de la meteorización de minerales sedimentarios, especialmente en áreas cercanas al mar Caribe.

Las sales pueden encontrarse en el suelo precipitadas bajo las formas de cristales, disueltas en la solución o retenidas y adsorbidas en el complejo de cambio. El contenido de sales en cualquiera de estas tres situaciones está cambiando continuamente debido a variaciones en la capacidad de humedad edáfica y al aporte que se pueda estar presentando en cada condición. Así, en el período seco la cristalización aumenta, por lo tanto la solución se concentra y aumentan las sales en la matriz del suelo; y en el período húmedo, el comportamiento es inverso. Según el IDEAM (2002) e Ibáñez et al. (2004), las sales más frecuentemente encontradas en los suelos son el $\mathrm{NaCl}, \mathrm{CaCl}_{2}, \mathrm{MgCl}_{2}, \mathrm{KCl}, \mathrm{MgSO}_{4}$, $(\mathrm{Na})_{2} \mathrm{SO}_{4}, \mathrm{NaNO}_{3}, \mathrm{KNO}_{3},(\mathrm{Na})_{2} \mathrm{CO}_{3}$ y $\mathrm{NaHCO}_{3}$.

D'Almeida et al. (2005) indican que el análisis de datos de salinidad deben ser realizados de modo que permita la identificación de factores que están contribuyendo en el aumento de las sales y de esta forma identificar las áreas afectadas, para implementar un monitoreo de las variaciones temporales, con el objetivo de planear el estudio de recuperación, definición de estrategias de manejo e identificación de los factores responsables del problema.

Al respecto Queiroz et al. (1997) indican que la utilización de la estadística clásica y de la geoestadística complementan el análisis e interpretación de datos de salinidad obtenidos en una determinada región. Regazzi y Leite (1992) y Maia et al. (2001) de igual forma exponen que la regresión lineal múltiple es una técnica usada ampliamente en la investigación agrícola, en especial en el área de suelos y aguas, sin embargo, debe emplearse solo en determinadas circunstancias para que el modelo estadístico usado represente de forma fiel el estudio analizado. Porque solo conociendo la verdadera relación entre variables independientes y dependiente se pueden hacer inferencias estadísticas precisas con utilidades prácticas para controlar y predecir las respuestas en el estudio de caso.

El procedimiento Stepwise es conocido como regresión por paso, debido a que reexamina en cada paso de la regresión la última variable que entra en el modelo, en relación con las variables ya incluidas en él, porque una variable ya incluida puede llegar a ser innecesaria y eliminarse del modelo al incorporar una nueva variable. Esto se prueba mediante una prueba $\mathrm{F}$ parcial para todas las variables del modelo, y si de todas ellas hay alguna que al entrar la última resulta ser no significativa, automáticamente queda eliminada del modelo que se está estimando (Rebolledo, 2002). Este trabajo fue realizado con el objetivo de desarrollar y validar modelos para la estimación de la conductividad eléctrica del extracto de saturación de suelos ubicados en desembocadura del río Sinú en el mar Caribe.

\section{Materiales y Métodos}

La zona de estudio (Figura 1) está compuesta por $82 \mathrm{~km}^{2}$ del área de influencia de la desembocadura del río Sinú, concentrada en los últimos $22 \mathrm{~km}$ de su recorrido y cuyos puntos extremos tienen coordenadas geográficas $9^{\circ} 27^{\prime} 22.3$ " de latitud $\mathrm{N} ; 75^{\circ} 56^{\prime} 34.5^{\prime \prime}$ de longitud W y 9०19'34.6” de latitud N; 7551'4.5" de longitud W. Esta zona limita al norte con el océano Atlántico, al sur con los corregimientos La Doctrina y Las Cañas, al este con las ciénagas de Ostional y al oeste por el complejo de lomeríos de José Manuel y Calle Ralita, en comprensión territorial de los municipios de San Bernardo del Viento y Lórica, del departamento de Córdoba, Colombia. De acuerdo con la clasificación de Holdridge (1978), esta área tiene como zona de vida la de bosque seco tropical (bs-T) con regímenes de precipitaciones promedio de $1.425 \mathrm{~mm}$ año ${ }^{-1}$, temperatura media de $27,6^{\circ} \mathrm{C}$ y humedad relativa del 84\% (Palencia et al., 2006).

Para el estudio se hicieron 10 muestreos de suelos correspondientes a lecturas tomadas en abril 2002, enero 2003, abril y noviembre de 2005; mayo y noviembre de 2006; abril y noviembre de 2007 y abril y noviembre del 2008. Cada muestreo comprendió 39 puntos de colecta de muestras ubicados próximos a pozos de observación, que se encontraban distribuidos en 25 pozos en vértices principales con espaciamiento de $2 \mathrm{~km}$ entre sí y 14 en vértices auxiliares con espaciamiento de $1 \mathrm{~km}$ respecto de los principales. En cada punto 


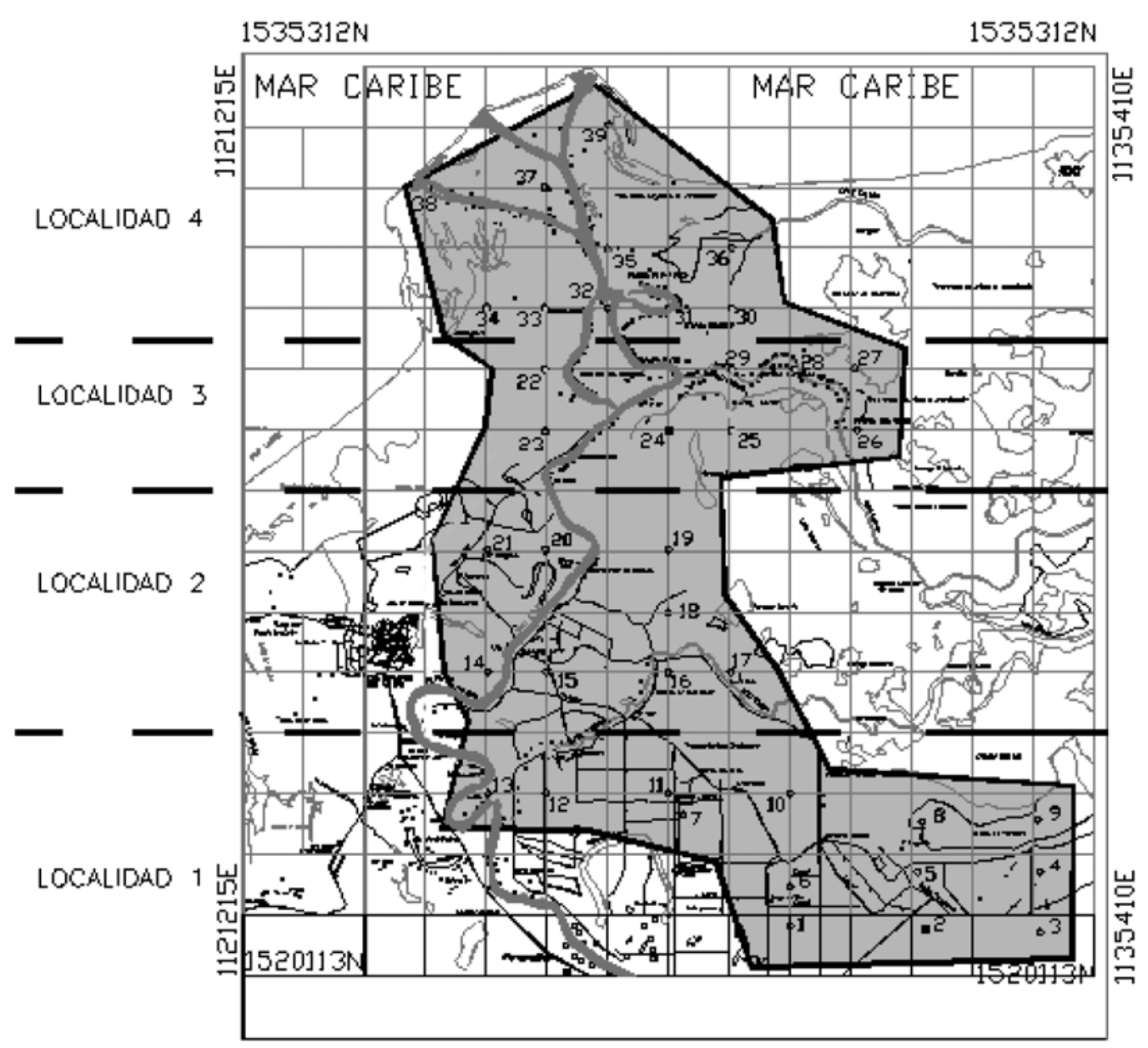

Figura 1. Ubicación de las localidades de la zona de estudio en los últimos $22 \mathrm{~km}$ del río Sinú en Córdoba, Colombia.

fueron colectados, además, 5 muestras de suelos a profundidades de $0-10,10-20,20-30,30-40$ y 40-50 cm, por pozo de observación.

Para la zona de estudio se definieron los periodos correspondientes al periodo seco y lluvioso del año, donde se analizó el registro histórico de precipitación de 16 años (1990 a 2005) de la Estación Meteorológica del municipio de San Bernardo Viento. Como periodo lluvioso se consideraron los meses que presentaron más del $10 \%$ de la precipitación media anual, que abarca el periodo de mayo a noviembre (más del $92 \%$ de la lluvia total), y por lo tanto el periodo seco correspondió al de diciembre a abril.

Las muestras de suelos fueron procesadas por el Laboratorio de Suelos y Aguas de la Universidad de Córdoba, según normas de Apha (1995) y en ellas se determinaron los siguientes parámetros: conductividad eléctrica en $\mathrm{dS} \mathrm{m}^{-1}(\mathrm{CE})$ por método conductimétrico, en un equipo Metrohm modelo 712 , cationes $\mathrm{Ca}^{2+}, \mathrm{Mg}^{2+}$ en cmol L ${ }^{-1}$ cuantificados por espectrofotometría de absorción atómica $\mathrm{Na}^{+} \mathrm{y}$ $\mathrm{K}^{+}$en cmol. $\mathrm{L}^{-1}$ por espectrofotometría de emisión atómica en un espectrofotómetro marca Pelkin 3110. Aniones $\mathrm{Cl}^{-}$en $\mathrm{cmol} \mathrm{L}^{-1}$ por titulación volumétrica con nitrato de plata $0.0141 \mathrm{~N}, \mathrm{HCO}_{3}^{-} \mathrm{y} \mathrm{CO}_{3}{ }^{2-} \mathrm{en} \mathrm{cmol}$ $\mathrm{L}^{-1}$ por titulación volumétrica con $\mathrm{H}_{2} \mathrm{SO}_{4}{ }^{2-} 0,02 \mathrm{~N}$ y $\mathrm{SO}_{4}{ }^{2-}$ en $\mathrm{cmol} \mathrm{L}^{-1}$ por el método turbidimétrico.

Los datos de $\mathrm{CE}$, cationes y aniones en periodos secos y lluviosos para las diferentes profundidades fueron sometidos a análisis de correlación simple de Pearson y estimación de modelos de regresión lineal múltiple por el método de regresión por pasos (The Stepwise regresión procedure), utilizando el 
programa estadístico SAS (Statistical Analysis System) v. 9.2.

La correlación simple contempló una matriz de correlación entre la CE y los iones determinados por el laboratorio en pasta de saturación y para la generación de los modelos, los iones $\mathrm{Ca}, \mathrm{Mg}$, $\mathrm{Na}, \mathrm{Cl}, \mathrm{K}, \mathrm{HCO}_{3}, \mathrm{CO}_{3}, \mathrm{y} \mathrm{SO}_{4}$ fueron las variables independientes y la $\mathrm{CE}$ la variable dependiente. La ecuación de regresión tuvo la siguiente forma:

$$
\mathrm{Y}=\mathrm{a}+\mathrm{bX} \mathrm{i}_{\mathrm{i}}+\mathrm{cX} \mathrm{i}_{\mathrm{i}+1}+\mathrm{dX}_{\mathrm{i}+2}+\mathrm{dX} \mathrm{X}_{\mathrm{i}+\mathrm{n}}+\mathrm{e}_{\mathrm{i}}
$$

Donde,

Y: conductividad eléctrica o variable dependiente, $\mathrm{dS} \cdot \mathrm{m}^{-1}$

$\mathrm{Xi}$ : variable independiente, $\mathrm{cmol} \cdot \mathrm{L}^{-1}$

a: constante de regresión

b, c, d: coeficientes angulares de la variable, y

e: error aleatorio

La contribución de las variables independientes que integraron los modelos se determinaron de acuerdo con el criterio propuesto por Maia et al. (2001), el que se basa en la magnitud de las sumas de cuadrados de estas variables.

\section{Validación de modelos}

Para la validación de la precisión de las ecuaciones de regresión obtenidas para las diferentes profundidades en periodos secos y lluviosos, se realizó la prueba de chi-cuadrado; donde la $\mathrm{CE}$ esperada correspondió a la estimada por los modelos de regresión lineal múltiple, y la CE observada a la registrada por el laboratorio para 20 puntos (19 al 39) de muestreo del área en estudio. Además se realizó correlación simple de Pearson entre las CE esperada y observada que fueron descritas anteriormente.

\section{Resultados y Discusión}

Las correlaciones lineales simples entre la CE y los iones estudiados en el extracto de saturación del suelo para las diferentes profundidades $0-10,10-20$, 20-30, 30-40 y 40-50 cm en el periodo seco y lluvioso se presentan en la Tabla 1. Para el periodo seco se observan correlaciones altamente significativas $(\mathrm{P}<0,01)$ entre la $\mathrm{CE}$ e iones salinizadores como $\mathrm{Cl}$, Na y $\mathrm{Mg}$ con $r \geq 0,76^{* *}, r \geq 0,75^{* *}$ y $r \geq 0,73^{* *}$, mientras que para el periodo lluvioso la CE presentan $r \geq 0,91 * *, r \geq 0,62 * * \mathrm{y} r \geq 0,53 * *$ con los iones $\mathrm{Cl}, \mathrm{Na}$ y Mg. La magnitud de estas correlaciones indica que para el periodo lluvioso se presenta un incremento en el grado de asociación de la $\mathrm{CE}$ con el ion $\mathrm{Cl}, \mathrm{y}$ una disminución de su asociación con los iones $\mathrm{Na}$ y $\mathrm{Mg}$ para profundidades superiores a los $20 \mathrm{~cm}$. Esta situación posiblemente se deba al aporte de cloruros provenientes de aguas freáticas con alta concentración de sales y la solubilidad de este tipo de sales presentes en estas condiciones. Además, Ruiz (1966) señala que en áreas de suelos como la región de La Doctrina y San Bernardo del VientoCórdoba, presentan antecedentes de depósitos de sales sepultadas, las cuales por proceso de

Tabla 1. Correlación simple (Pearson) entre CE e iones analizados en el extracto de saturación para diferentes profundidades en periodos lluviosos y secos en el área de influencia de la desembocadura del río Sinú, Córdoba, Colombia.

\begin{tabular}{|c|c|c|c|c|c|c|c|c|}
\hline \multicolumn{9}{|c|}{ Periodo Seco: Diciembre -Abril } \\
\hline Profundidades & & $\mathrm{Ca}^{2+}$ & $\mathrm{Mg}^{2+}$ & $\mathrm{Na}^{+}$ & $\mathrm{K}^{+}$ & $\mathrm{Cl}^{-}$ & $\mathrm{HCO}_{3}{ }^{2-}$ & $\mathrm{SO}_{4}{ }^{2-}$ \\
\hline \multicolumn{9}{|l|}{$\mathrm{cm}$} \\
\hline $0-10$ & $\mathrm{CE}$ & $0,54 * *$ & $0,81 * *$ & $0,78 * *$ & $0,31 *$ & $0,77 * *$ & $0,11 \mathrm{~ns}$ & $0,22 * *$ \\
\hline $0-20$ & & $0,44 * *$ & $0,79 * *$ & $0,83 * *$ & $0,69 * *$ & $0,93 * *$ & $0,10 \mathrm{~ns}$ & $0,16^{*}$ \\
\hline $20-30$ & & $0,71 * *$ & $0,92 * *$ & $0,75^{* *}$ & $0,46 * *$ & $0,76^{* *}$ & $0,05 \mathrm{~ns}$ & $0,21 *$ \\
\hline $30-40$ & & $0,30 * *$ & $0,73^{* *}$ & $0,85^{* *}$ & $0,50 * *$ & $0,91 * *$ & $0,04 \mathrm{~ns}$ & $0,14 \mathrm{~ns}$ \\
\hline $40-50$ & & $0,43 * *$ & $0,84 * *$ & $0,86^{* *}$ & $0,39 * *$ & $0,92 * *$ & $0,07 \mathrm{~ns}$ & $0,33 * *$ \\
\hline \multicolumn{9}{|c|}{ Periodo lluvioso: mayo-noviembre } \\
\hline $0-10$ & $\mathrm{CE}$ & $0,71 * *$ & $0,84 * *$ & $0,74 * *$ & $0,41 * *$ & $0,91 * *$ & $0,13 \mathrm{~ns}$ & $0,55^{* *}$ \\
\hline $0-20$ & & $0,76^{* *}$ & $0,84 * *$ & $0,73 * *$ & $0,42 * *$ & $0,95^{* *}$ & $0,16 \mathrm{~ns}$ & $0,52 * *$ \\
\hline $20-30$ & & $0,77 * *$ & $0,79 * *$ & $0,75^{* *}$ & $0,55^{* *}$ & $0,94 * *$ & $0,19 *$ & $0,65^{* * *}$ \\
\hline $30-40$ & & $0,58 * *$ & $0,53^{* *}$ & $0,62 * *$ & $0,41 * *$ & $0,95^{* *}$ & $0,16 \mathrm{~ns}$ & $0,48^{* *}$ \\
\hline $40-50$ & & $0,69 * *$ & $0,78^{* *}$ & $0,70^{* *}$ & $0,56^{* *}$ & $0,91 * *$ & $0,19 *$ & $0,61 * *$ \\
\hline
\end{tabular}

*significativo (5\%), **altamente significativo (1\%), ns: no significativo. 
capilaridad pueden llegar a la superficie y producir alta salinización en la superficie de los suelos, lo que incrementa la presión osmótica en suelos y agua. Según Porta et al. (1999) valores muy altos de la presión osmótica en el agua del suelo, interfiere en el crecimiento de la mayoría de los cultivos y otras plantas no especializadas.

De igual forma en condiciones de escaso drenaje de estas zonas próximas al mar, donde se presentan suelos arcillosos compactados y son zonas de ganancias por su posición geomorfológica entre otros, son condiciones que favorecen la acumulación de las sales en suelos y aguas, por lo tanto para el manejo edafológico de estas áreas agrícolas se debe implementar la construcción de canales de drenaje que intercepten el ascenso capilar de las aguas freáticas que traen consigo altas concentraciones de iones salinizadores como el $\mathrm{Cl}$.

En términos generales, los modelos generados presentan entre 2 y 4 parámetros significativos y altamente significativos, donde la contribución del ion $\mathrm{Cl}$ fue predominante en casi todas las profundidades en los dos periodos de estudio. Otros iones que aportaron a los modelos de CE fueron el $\mathrm{Na}, \mathrm{Mg}$, $\mathrm{Ca}, \mathrm{K} \mathrm{y} \mathrm{SO}_{4}$ (Tabla 2). Estos modelos de regresión lineal múltiple presentaron para la $\mathrm{CE}$ coeficientes de determinación $\left(\mathrm{R}^{2}\right)$ entre 0,84 y 0,96 en las diferentes profundidades para el periodo seco y entre 0,87 y 0,93 en el periodo lluvioso, además con el análisis de los resultados mediante la correlación de Pearson se observa que existe una considerable correlación entre los iones de los modelos y la $\mathrm{CE}$, indicando el alto grado de confiabilidad que presentan estas ecuaciones para estimar los valores de CE en función de la concentración de los iones implicados en los modelos. Estos resultados son similares a los reportados por D'Almeida et al. (2005), los cuales obtuvieron modelos de CE para diferentes profundidades con $\mathrm{R}^{2}$ entre $0,87 \mathrm{y}$ 0,99 para los municipios de Limoeiro do Norte y Quixeré del estado Ceará en Brasil, y los iones que integraron los modelos fueron el $\mathrm{Cl}, \mathrm{Na}, \mathrm{Mg}$ y Ca. Estos autores encontraron coeficientes de correlación superiores a 0,97 y desvío de la inclinación de la recta inferiores a 3,6\% e indican que estos parámetros demuestran alta precisión y exactitud, asimismo indican que el modelo no es tendencioso, porque estima valores bien próximos del real, para altas o bajas concentraciones de sales.

En el periodo seco en los primeros $20 \mathrm{~cm}$ de profundidad del suelo, los iones que contribuyen en la generación de los modelos son el $\mathrm{Ca}, \mathrm{Cl}, \mathrm{Na}, \mathrm{Mg}$ y $\mathrm{SO}_{4}$, mientras que en profundidades superiores a los $20 \mathrm{~cm}$, los iones $\mathrm{Cl}, \mathrm{Na}$ y $\mathrm{Mg}$ aparecen principalmente contribuyendo en los modelos de CE. Esta relación es correspondiente a la magnitud de las correlaciones simples para este periodo, en donde profundidades superiores a los $20 \mathrm{~cm}$ presentaron los coeficientes de correlación $(r)$ más altos para los iones $\mathrm{Cl}$, Na y $\mathrm{Mg}$. Para el periodo lluvioso los modelos indican una mayor participación de los iones $\mathrm{K}_{\text {y }} \mathrm{SO}_{4} \mathrm{los}$ que presentan una mayor correlación con la $\mathrm{CE}$ en relación con el periodo seco (Tablas 1 y 2). Es importante indicar que en estas condiciones se presentaron algunos parámetros negativos en los modelos, condición que puede obedecer posiblemente

Tabla 2. Modelos de regresión lineal múltiple, coeficientes de determinación y contribución de las variables independientes para la estimación de la CE del suelo en el área de influencia de la desembocadura del río Sinú en periodos lluviosos y secos.

\begin{tabular}{|c|c|c|c|c|}
\hline $\operatorname{Pr}(\mathrm{cm})^{11}$ & Ecuación de regresión lineal múltiple & $\mathrm{R}^{2}$ & $\mathrm{~F}$ & Contribución \\
\hline \multicolumn{5}{|c|}{ Periodo seco: diciembre-abril } \\
\hline $0-10$ & $\mathrm{CE}=0,20792+0,2293 \mathrm{Ca}^{* *}+0,02280 \mathrm{Na}^{* *}+0,04233 \mathrm{Cl} * *+0.0281 \mathrm{SO}_{4}^{* *}$ & 0,8527 & $<0.0001$ & $\mathrm{Ca}>\mathrm{Cl}>\mathrm{SO}_{4}>\mathrm{Na}$ \\
\hline $0-20$ & $\mathrm{CE}=0,54533+0,0628 \mathrm{Mg} * *+0,03992 \mathrm{Na} * *+0,05320 \mathrm{Cl} * *-0.0288 \mathrm{SO}_{4}^{* *}$ & 0,9362 & $<0.0001$ & $\mathrm{Cl}>\mathrm{Na}>\mathrm{Mg}>\mathrm{SO}_{4}$ \\
\hline $20-30$ & $\mathrm{CE}=0,05656+0,1482 \mathrm{Mg} * *+0,02258 \mathrm{Na} * *+0,04268 \mathrm{Cl} * *$ & 0,8492 & $<0.0001$ & $\mathrm{Cl}>\mathrm{Mg}>\mathrm{Na}$ \\
\hline $30-40$ & $\mathrm{CE}=0,57633+0,0284 \mathrm{Na}^{* *}+0,06832 \mathrm{Cl} * *$ & 0,8538 & $<0.0001$ & $\mathrm{Cl}>\mathrm{Na}$ \\
\hline $40-50$ & $\mathrm{CE}=0,15739+0,1571 \mathrm{Mg} * *+0,02593 \mathrm{Na}^{* *}+0,04063 \mathrm{Cl}^{* *}$ & 0,9686 & $<0.0001$ & $\mathrm{Cl}>\mathrm{Mg}>\mathrm{Na}$ \\
\hline \multicolumn{5}{|c|}{ periodo lluvioso: mayo-noviembre } \\
\hline $0-10$ & $\mathrm{CE}=0,75333+0,06401 \mathrm{Na}^{* *}+0,07665 \mathrm{~K} * *+0,05499 \mathrm{Cl} * *-0,06714 \mathrm{SO}_{4} * *$ & 0,8739 & $<0.0001$ & $\mathrm{Cl}>\mathrm{Na}>\mathrm{SO}_{4}>\mathrm{K}$ \\
\hline $0-20$ & $\mathrm{CE}=0,77034-0,07280 \mathrm{Mg}^{*}-0,03134 \mathrm{~K}^{* *}+0,12033 \mathrm{Cl} * *$ & 0,9167 & $<0.0001$ & $\mathrm{Cl}>\mathrm{K}>\mathrm{Mg}$ \\
\hline $20-30$ & $\mathrm{CE}=0,65705+0,03349 \mathrm{Na} * *+0,09780 \mathrm{Cl} * *-0,06615 \mathrm{SO}_{4} * *$ & 0,9324 & $<0.0001$ & $\mathrm{Cl}>\mathrm{Na}>\mathrm{SO}_{4}$ \\
\hline $30-40$ & $\mathrm{CE}=0,66647-0,03427 \mathrm{Mg}^{* *}-0,02433 \mathrm{~K}^{* *}+0,11166 \mathrm{Cl}^{* *}$ & 0,9125 & $<0.0001$ & $\mathrm{Cl}>\mathrm{Mg}>\mathrm{K}$ \\
\hline $40-50$ & $\mathrm{CE}=0,78661+0,05243 \mathrm{Na}^{* *}+0,04489 \mathrm{~K}^{* *}+0,05277 \mathrm{Cl}^{* *}$ & 0,8809 & $<0.0001$ & $\mathrm{Cl}>\mathrm{Na}>\mathrm{K}$ \\
\hline
\end{tabular}

${ }^{/ 1} \mathrm{Pr}$ : Profundidad $\mathrm{CE}=$ Conductividad eléctrica en $\mathrm{dS} \mathrm{m}{ }^{-1} \mathrm{Ca}, \mathrm{Mg}, \mathrm{Na}, \mathrm{K}, \mathrm{Cl}, \mathrm{CO}_{3}, \mathrm{SO}_{4}$ en $\mathrm{cmol} \mathrm{L}^{-1}$.

*significativo (5\%), **altamente significativo $(1 \%) \mathrm{R}^{2}$ : coeficiente de determinación. 
a factores como la solubilidad de los iones, tipos de iones, radio de hidratación y la disponibilidad en agua y precipitación de compuestos insolubles, que influyen en la formación de los compuestos de variada solubilidad que intervienen en la $\mathrm{CE}$ del suelo. Hasegawa et al. (2000) exponen que los cationes estrechamente relacionados con la salinidad son $\mathrm{Na}^{+}, \mathrm{Ca}^{2+} \mathrm{y} \mathrm{Mg}^{2+}$, mientras que $\mathrm{Cl}^{-}$, sulfato $\left(\mathrm{SO}^{2-}\right)$ y bicarbonato $\left(\mathrm{HCO}_{3}^{-}\right)$son los aniones que contribuyen a la salinidad del suelo. Ridd y Stieglitz (2002) determinaron que los rápidos aumentos de salinidad en estuarios en las partes áridas de Australia fueron en gran parte el resultado de la evaporación.

Berner (1971) indica que la reducción de sulfatos en agua de mar incrementan la alcalinidad, resultando en la precipitación de diferentes carbonatos, además la CE es influenciada por el contenido de agua, el contenido de arcilla y la presencia de iones intercambiables en el suelo Corwin et al. (1999). Queiroz et al. (2010) exponen que en los suelos salinos las propiedades químicas y físicas tienen gran variabilidad espacial y temporal, debido a las influencias de las prácticas de manejo utilizadas, profundidad del nivel freático, permeabilidad del suelo, tasa de evapotranspiración, lluvias, salinidad del agua subterránea y otros factores geohidrológicos.

Las Figuras 2 y 3 representan la comparación de los valores de CE estimadas por los modelos de regresión lineal múltiple y la determinada por laboratorio en periodos lluviosos y secos. Para todas las profundidades y en los dos periodos evaluados la prueba de chi-cuadrado no reflejó diferencias significativas entre los valores de CE, adicionalmente la correlación entre esos valores fue altamente significativa y con magnitud entre 0,96 y 0,99 para el periodo seco y entre 0,86 y 0,98 para el periodo lluvioso.

Estos valores indican una alta asociación, precisión y correspondencia entre los valores de CE estimadas por los modelos de la regresión y la dada por el laboratorio. Si la correlación entre las variables son significativas, dan como resultado predicciones locales más exactas (Triantafilis et al., 2001). D'Almeida et al. (2005) utilizando modelos de regresión lineal múltiple para la estimación de la CE para diferentes profundidades de suelos, encontraron modelos con coeficientes de correlación entre 0,94 y 0,99 y resaltan que los iones seleccionados como variables independientes mostraron alta correlación con la CE estimada. El análisis de regresión en estudios de concentración de iones y la conductividad eléctrica estimada viene siendo utilizada con suceso por varios investigadores como Nunes Filho et al. (2000). Mandelbrot (2002) expone que el uso de sensores remotos y las ventajas de las técnicas estadísticas, así como la aplicación de los modelos matemáticos desarrollados para el análisis de los sistemas complejos, son una alternativa variable para estudiar la salinidad de los suelos a diferentes escalas.

En la Tabla 3 se presentan las diferencias medias absolutas entre los valores de CE estimadas por los modelos de regresión y la obtenida por laboratorio para los periodos seco y lluvioso a diferentes profundidades. Para el periodo seco estas diferencias absolutas fueron de 0,14 a $0,56 \mathrm{dS} \mathrm{m}^{-1}$ a nivel de profundidades, enmarcados dentro de la categoría de suelos no salinos $\left(\mathrm{CE}<2 \mathrm{dS} \mathrm{m}^{-1}\right)$, de 0,23 a $1,14 \mathrm{dS} \mathrm{m}^{-1}$ para suelos ligeramente salinos $\left(\mathrm{CE}=2\right.$ a $\left.4 \mathrm{dS} \mathrm{m}^{-1}\right)$, de 0,56 a $1,83 \mathrm{dS} \mathrm{m}^{-1}$ para suelos medianamente salinos $\left(\mathrm{CE}=4 \mathrm{a} 8 \mathrm{dS} \mathrm{m}^{-1}\right)$, de 1,1 a 4,3 $\mathrm{dS} \mathrm{m}^{-1}$ para suelos fuertemente salinos $\left(\mathrm{CE}=8 \mathrm{a} 16 \mathrm{dS} \mathrm{m}^{-1}\right)$ y de 1,64 a $4,36 \mathrm{dS} \mathrm{m}^{-1}$ para suelos extremadamente salinos $\left(\mathrm{CE}=>16 \mathrm{dS} \mathrm{m}^{-1}\right)$. Estos valores indican que la precisión de la $\mathrm{CE}$ estimada por los modelos disminuye a medida que incrementa la salinidad de los suelos. D'Almeida et al. (2005) encontraron que cuando los valores de CE estimada son superiores a $1 \mathrm{dS} / \mathrm{m}^{-1}$ el modelo muestra tendencia a subestimar la CE real, lo que sugiere que para valores elevados de $\mathrm{CE}$ esperada ocurre una interferencia de otros iones con intensidad mayor.

Sin embargo, en términos prácticos y operativos, la mayor precisión de los modelos es requerida en las primeras dos categorías de clasificación de suelos salinos, ya que estos valores determinan la presencia de problemas de salinidad de los suelos. Según Allaire et al. (2012), estas propiedades están claramente asociadas con la salinidad del suelo y su estimación y predicción espacial representan un especial interés científico, para nuevas aplicaciones agrícolas o ambientales; y Parr et al. (1992) indican que diferentes atributos químicos, físicos y biológicos interactúan de manera compleja y su entendimiento es esencial para favorecer la sostenibilidad y mejorar la capacidad productiva del suelo. Shao et al. (2012) manifiestan que la salinidad del suelo, definida como la concentración de sal mineral soluble que existe en el suelo, es uno de los factores ambientales más severos que limitan la productividad de los cultivos agrícolas. 


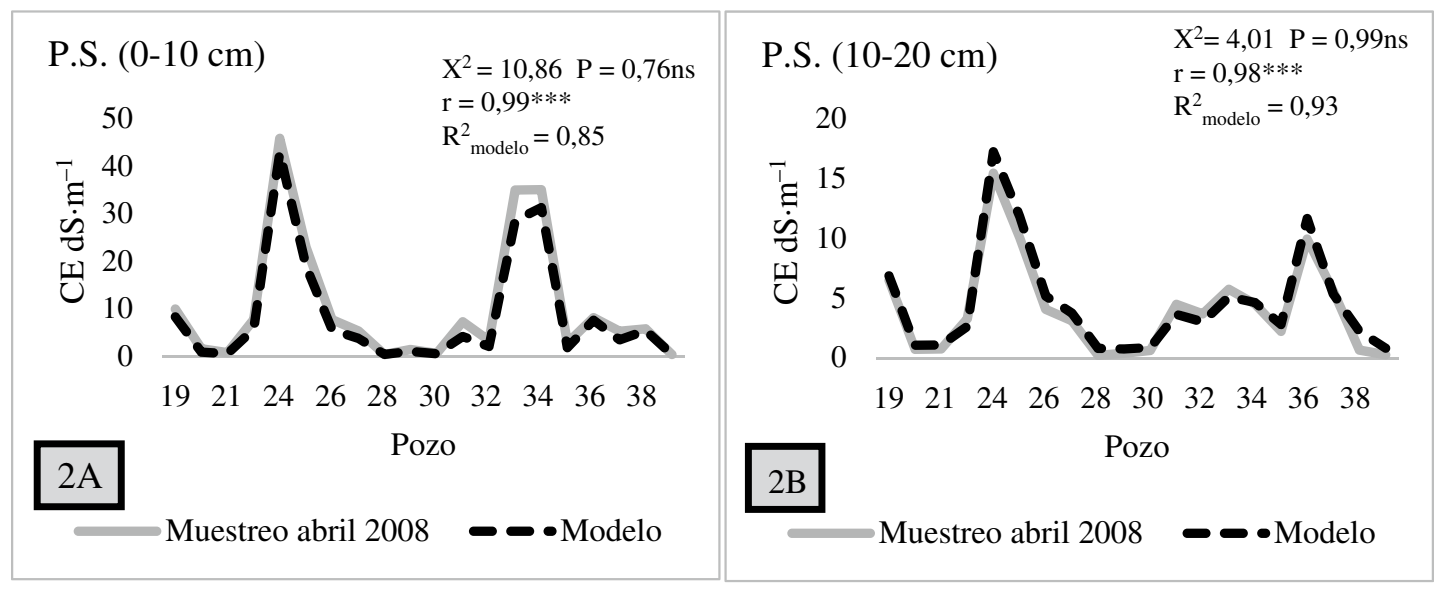

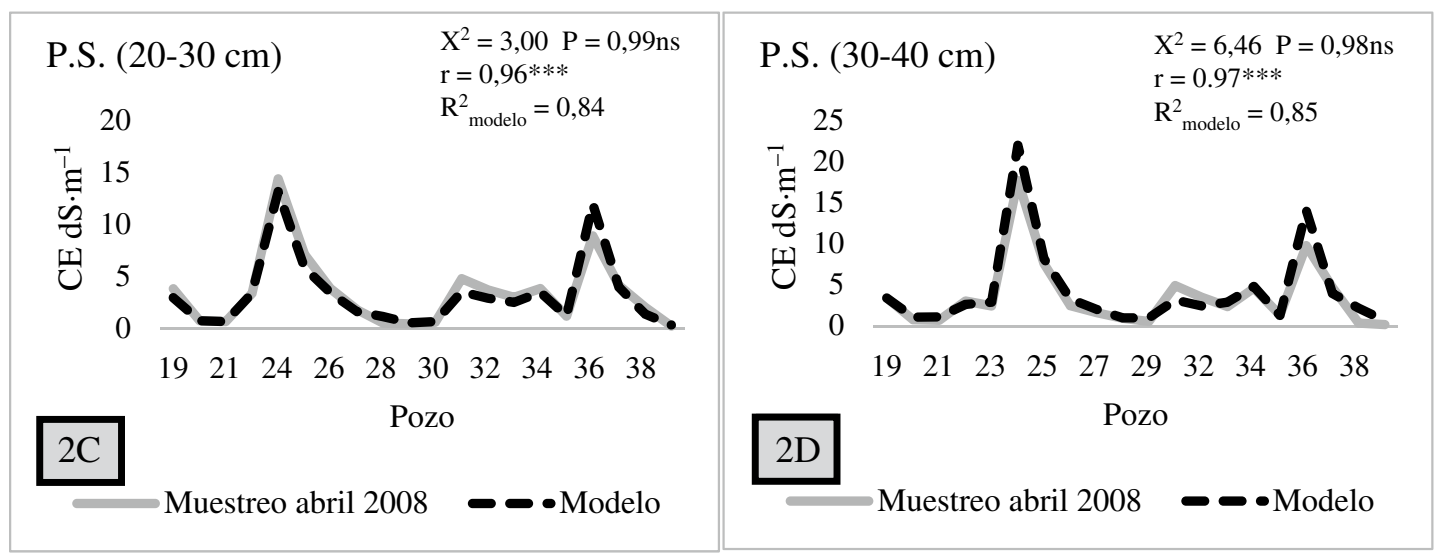

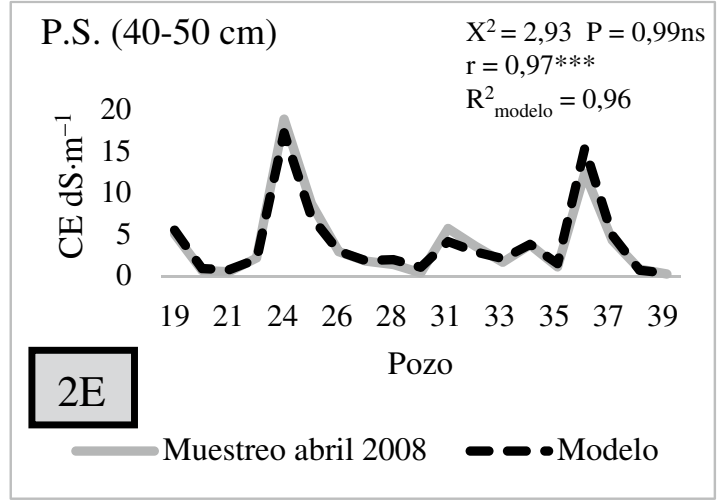

Figura 2. Comparación de la CE estimada por los modelos y la obtenida en laboratorio en muestreo de abril de 2008 (periodo seco) en pozos del 19 al 39 del área de estudio. $X^{2}$ : chi-cuadrado; $P=$ probabilidad de chi-cuadrado; ns: no significativo; $r=$ coeficiente de correlación simple de Pearson; $\mathrm{R}_{\text {modelo }}^{2}=$ Coeficiente de determinación del modelo de $\mathrm{CE}$ calculado por la regresión lineal múltiple; *** altamente significativo.

Es importante señalar que las dos primeras clasificaciones de suelos no salinos y ligeramente salinos representan entre el $40 \%$ y $75 \%$ de los puntos de muestreo validados y son los que presentan los rangos más estrechos, lo que hace pertinente que el mejor ajuste de los modelos esté sobre estas dos clasificaciones. De igual forma, las clasificaciones de suelos moderada a extremadamente salinos presentan rangos más amplios y a su vez, por sí mismas, representan un problema de salinización 

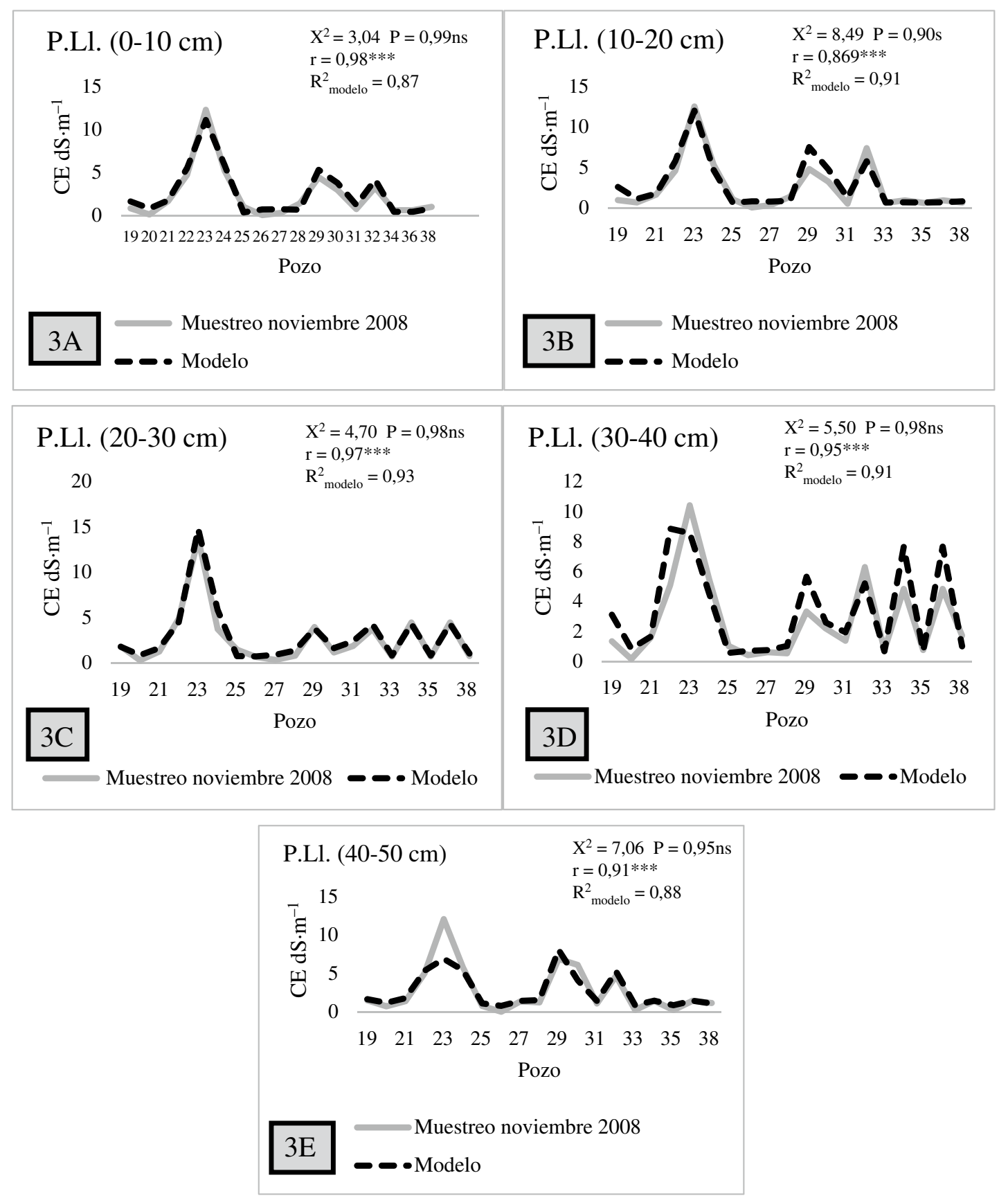

Figura 3. Comparación de la CE estimada por los modelos y la obtenida en laboratorio en muestreo de noviembre de 2008 (periodo lluvioso) en pozos del 19 al 39 del área de estudio. $X^{2}$ : chi-cuadrado; $P=$ probabilidad de chi-cuadrado; ns: no significativo; $r=$ coeficiente de correlación simple de Pearson; $\mathrm{R}^{2}$ modelo $=$ Coeficiente de determinación del modelo de CE calculado por la regresión lineal múltiple; *** altamente significativo. 
Tabla 3. Promedio de diferencias absolutas $\left(\mathrm{dS} \mathrm{m}^{-1}\right)$ entre CE estimadas por los modelos de regresión lineal múltiple y las obtenidas por laboratorio de acuerdo con la clasificación de suelos salinos.

\begin{tabular}{lccccc}
\hline \multirow{2}{*}{$\operatorname{Pr}(\mathrm{cm})$} & $<2$ & 02 -abr & 04-ago & ago-16 & $>16$ \\
\cline { 2 - 6 } & \multicolumn{5}{c}{ CE $\left(\mathrm{dS} \cdot \mathrm{m}^{-1}\right)$} \\
\hline \multicolumn{2}{l}{ Periodo seco: } & diciembre -abril & & & \\
$0-10$ & $0,23(30 \%)^{+}$ & $1,14(10 \%)$ & $1,83(30 \%)$ & $1,10(10 \%)$ & $4,36(20 \%)$ \\
$10-20$ & $0,56(35 \%)$ & $0,63(20 \%)$ & $0,56(30 \%)$ & $1,76(15 \%)$ & - \\
$20-30$ & $0,14(40 \%)$ & $0,51(35 \%)$ & $0,91(15 \%)$ & $2,15(10 \%)$ & - \\
$30-40$ & $0,49(40 \%)$ & $0,55(30 \%)$ & $0,82(20 \%)$ & $4,30(5 \%)$ & $4,28(5 \%)$ \\
$40-50$ & $0,31(47 \%)$ & $0,23(21 \%)$ & $0,89(16 \%)$ & $2,30(11 \%)$ & $1,64(5 \%)$ \\
\hline \multicolumn{2}{l}{ Periodo lluvioso: mayo-noviembre } & & & \\
$0-10$ & $0,37(63,2 \%)$ & $0,94(15,8 \%)$ & $0,24(15,8 \%)$ & $1,04(5,3 \%)$ & - \\
$10-20$ & $0,48(57,9 \%)$ & $1,37(10,5 \%)$ & $2,29(26,3 \%)$ & $1,85(5,3 \%)$ & - \\
$20-30$ & $0,46(64,7 \%)$ & $0,86(11,8 \%)$ & $0,76(17,6 \%)$ & $1,18(5,9 \%)$ & - \\
$30-40$ & $0,45(68,4 \%)$ & $1,53(5,3 \%)$ & $1,53(21,1 \%)$ & $0,51(5,3 \%)$ & - \\
$40-50$ & $0,32(68,4 \%)$ & - & $0,88(26,3 \%)$ & $5,14(5,3 \%)$ & - \\
\hline
\end{tabular}

$\mathrm{CE}<2$ : suelos no salinos, 2-4: ligeramente salinos, 4-8: medianamente salinos, 8-16: fuertemente salinos, $>16$ : extremadamente salinos. Pr: profundidad ${ }^{+}$Valores en paréntesis hacen referencia al porcentaje de pozos dentro de cada rango de clasificación de suelos por salinidad.

de suelos, por lo que el sesgo para estas categorías puede ser más permisivo y/o aceptable. APHA (1992) indica que la determinación del contenido total de sales requiere de análisis químicos que consumen mucho tiempo, y para esto se pueden utilizar métodos indirectos para estimar la salinidad.

Para el periodo lluvioso en suelos no salinos las diferencias medias absolutas de CE no alcanzaron los $0,5 \mathrm{dS} \mathrm{m}^{-1}$, mientras que para suelos ligeros, medianos y fuertemente salinos fueron entre 0,86 y $1,37 \mathrm{dS}$ $\mathrm{m}^{-1}$, de 0,24 a $2,29 \mathrm{dS} \mathrm{m}^{-1}$ y de 0,51 a $5,14 \mathrm{dS} \mathrm{m}^{-1}$, respectivamente. Para este periodo ningún punto de muestreo se enmarcó dentro de la categoría de suelos extremadamente salinos. De igual forma, los suelos no salinos y ligeramente salinos representaron entre el $68,4 \%$ y $79 \%$ de los puntos muestreados, mientras que los suelos fuertemente salinos correspondieron entre el 5,3 y 5,9\% de estos puntos muestreados (Tabla 3 ). Resultados que son importantes de conocer, porque de acuerdo con Banin and Fish (1995) la salinización disminuye el crecimientos de los cultivos, incrementa la pérdida de suelos por erosión y contribuye con la desertificación.

En términos generales, el periodo seco presentó mayores valores de CE en comparación con el periodo lluvioso para los 20 puntos de muestreo, siendo las profundidades de $0-10 \mathrm{~cm}$ y de $10-20 \mathrm{~cm}$ las de máxima $\mathrm{CE}$, posiblemente producto de los procesos de evapotranspiración que contribuyen en el aumento de la salinidad de los suelos.

\section{Conclusiones}

La generación y validación de modelos de regresión lineal múltiple permiten realizar estimaciones muy próximas y confiables de la CE para el periodo seco y lluvioso del área de influencia de la desembocadura del río Sinú.

Los modelos estimados permitieron conocer la influencia, contribución y distribución de los iones salinizadores $\mathrm{Cl}, \mathrm{Na}$ y $\mathrm{Mg}$, principalmente en el perfil del suelo.

Los valores de CE varían con la profundidad de los suelos y los regímenes de precipitación, siendo mayor en el periodo seco y sobre los primeros $20 \mathrm{~cm}$.

Los modelos obtenidos para las profundidades de $10-20 \mathrm{~cm}$ y $40-50 \mathrm{~cm}$ para el periodo seco y de $10-20 \mathrm{~cm}, 20-30 \mathrm{~cm}$ y $30-40 \mathrm{~cm}$ en el periodo lluvioso presentan las mejores simulaciones y los más altos coeficientes de determinación $\left(\mathrm{R}^{2} \geq 0,91\right)$ y correlación $\left(r \geq 0,86^{* *}\right)$.

\section{Agradecimientos}

Al proyecto de investigación "Diagnóstico general del nivel de salinización en la zona aledaña a la desembocadura del río Sinú y su impacto socioeconómico: Período abril/2002noviembre/2008" y personal técnico del Laboratorio de Suelos y Aguas de la Universidad de Córdoba. 


\section{Literatura Citada}

Allaire, S.E.; Lange, S.F.; Lafond, J.A.; Pelletier, B.; Cambouris, A.N.; Dutilleul, P

2012. Multiscale spatial variability of $\mathrm{CO}_{2}$ emissions and correlations with physico-chemical soil properties. Geoderma, (Netherlands). 170: 251-260.

American Public Health Association (Apha)

1995. Standard methods for examination of water and wastewater. 19th Edition. Washington DC. 380 pp.

American Public Health Association

1992. Standard Methods for the examination of Water and Wastewater. 18th Edition. Washington, APHA, pp. 2-43.

Banin, A. and Fish, A.

1995. Secondary desertification due to salinization of intensively irrigated lands: The Israeli experience, Environ. Monit. Assess, 37: 17-37.

Berner, R.A.

1971. Principles of chemical Sedimentology: New York, McGraw-Hill, 240 pp.

Corwin, D.L.; Loague, K.; Ellsworth, T.R.

1999. Introduction: Assessing non-point source pollution in the vadose zone with advanced information technologies. In: Corwin, D.L.; Loague, K.; Ellsworth, T.R. eds. Assessment of Nonpoint Source Pollution in the Vadose Zone. Geophysical Monograph Series. Vol. 108. AGU, Washington, D.C., USA, pp. 1-20.

D’Almeida, D.; Andrade, E., Meireles, A.; Ness, R.

2005. Importância relativa dos íons na salinidade de um Cambissolo na Chapada do Apodi, Ceará. Eng. Agríc., Jaboticabal, 25 (3): 615-621.

Hasegawa, P,M.; Bressan, R.A; Zhu. J-K.; Bohnert, H.J.

2000. Plant cellular and molecular responses to high salinity. Annual Review of Plant Physiology and Plant Molecular Biology 51, 463-499.

Holdridge, R.

1978. Ecología basada en zonas de vida. IICA Libros y Materiales Educativos, 34.

\section{IDEAM}

2002. Zonificación de los procesos de salinización de los suelos de Colombia. Instituto de Hidrología, Meteorología y Estudios Ambientales-IDEAM, Subdirección de Geomorfología y Suelos. 44 pp.

Ibáñez, C.; Paloqueme, S.; Fontúrbel, F.

2004. Elementos principales del suelo, geodinámica y dinámica de los principales componentes del suelo. En: Fontúrbel, F. Ibáñez, C y Abruzzese (eds.) El Recurso Suelo: Bases edafológicas, problemática, administración y contaminación.

Maia, C.E.; Morais, E.R.C. de; Oliveira, M.

2001. Classificação da composiçãoiônica da agua de irrigação usando regressão linear múltipla. Revista Brasileira de Engenharia Agrícola e Ambiental, 5 (1): 55-59.
Mandelbrot, B.B.

2002. Gaussian Self-Affinity and Fractals. Springer Verlag. Berlin. 654 pp.

Nunes Filho, J.; Sousa, R. de; SÁ, V.A. de L.; Lima, B.P. 2000. Relações entre a concentração de águas subterrâneas e superficiais, visando à irrigação, no sertão de Pernambuco. Revista Brasileira de Engenharia Agrícola e Ambiental, Campina Grande, 4 (2): 189-93.

Palencia, G.; Mercado, T.; Combatt, E.

2006. Estudio agroclimático del departamento de Córdoba. Facultad de Ciencias Agrícolas, Universidad de Córdoba. $126 \mathrm{pp}$.

Parr, J.F.; Hornick, S.B.; Papendick, R.I.

1992. Soil quality: attributes and relationship to alternative and sustainable agriculture. Amer. J. Alternative Agric. 7 (1-2): 5-1.

Porta C.; López-Acevedo R.; Roquero de L.

1999. Edafología. Mundi-Prensa, Madrid, pp. 454; 657-705.

Queiroz, J.; Gonçalves, A.; Souto, J.; Folegatti, M.

1997. Avaliação e monitoramento da salinidade do solo. Em: Gheyi, H.R.; Queiroz, J.E.; Medeiros, J.F. (Ed.) Manejo e controle da salinidade na agricultura irrigada. Campina Grande: UFPB. pp. 69-111.

Queiroz, J. E.; Gonçalves, A. C. A.; Souto, J. S.; Folegatti, M. V. 2010. Avaliação e monitoramento da salinidade do solo. In: Gheyi, H. R.; Dias, N. da S.; Lacerda, C. F. Manejo da salinidade na agricultura. Fortaleza: INCT Sal. 472 pp.

Rebolledo, $\mathrm{H}$

2002. Manual SAS para computadora: análisis estadísticos de datos experimentales. Ed. Trillas, Mexico. 208 pp.

Regazzi, A., Leite, H.

1992. Análise de regressão: Teoria à aplicaçãoem manejo florestal. Viçosa, MG. 236 pp.

Ridd, P. V. and Stieglitz, T.

2002. Dry season salinity changes in arid estuaries fringed by mangroves and saltflats. Estuarine Coastal and Shelf Science, 54: 1039-1049

Ruiz, B.

1966. Estudio detallado de suelos y clasificación de tierras para riego y drenaje "La Doctrina". IGAC-INCORA. Bogotá, D.E. 173 pp.

Shao, X. H.; Hou, M. M.; Chena, L.H.;Changa, T.T.; Wanga, W.N. 2012. Evaluation of Subsurface Drainage Design Based on Projection Pursuit, International Conference on Future Energy, Environment, and Materials, Procedia,16: 747-752.

Triantafilis, J.; Huckel, A.I.; Odeh, I.O.A.

2001. Comparison of statistical prediction methods for estimating fieldscale clay content using different combinations of ancillary variables. Soil Sci. 166, 415-427. 